\title{
State Resolved Vibrational Relaxation Modeling for Strongly Nonequilibrium Flows
}

\author{
Iain D. Boyd* \\ Department of Aerospace Engineering, University of Michigan, Ann Arbor, MI, 48109 \\ Eswar Josyula ${ }^{\dagger}$ \\ U.S. Air Force Research Laboratory, Wright-Patterson Air Force Base, OH, 45433
}

\begin{abstract}
Vibrational relaxation is an important physical process in hypersonic flows. Activation of the vibrational mode affects the fundamental thermodynamic properties and finite rate relaxation can reduce the degree of dissociation of a gas. Low fidelity models of vibrational activation employ a relaxation time to capture the process at a macroscopic level. High fidelity, state-resolved models have been developed for use in continuum gas dynamics simulations based on Computational Fluid Dynamics (CFD). By comparison, such models are not as common for use with the direct simulation Monte Carlo (DSMC) method. In this study, a high fidelity, state-resolved vibrational relaxation model is developed for the DSMC technique. Results obtained for integrated rate coefficients from the DSMC model are consistent with the corresponding CFD model. Comparison of relaxation results obtained with the high-fidelity DSMC model show significantly less excitation of upper vibrational levels in comparison to the standard, lower fidelity DSMC vibrational relaxation model. Application of the new DSMC model to a Mach 7 normal shock wave in carbon monoxide provides better agreement with experimental measurements than the standard DSMC relaxation model.
\end{abstract}

\section{Introduction}

$\mathrm{V}$ ibrational relaxation is an important physical process in hypersonic flows due to the high temperatures that are generated. For air molecules, when temperatures exceed about 1,000 K, the vibrational mode becomes significantly activated leading to important changes in fundamental thermodynamic properties such as the ratio of specific heats. At low pressure flow conditions, the rate of vibrational activation is finite, and the gas lies in a state of thermal nonequilibrium with different temperatures associated with the translational and vibrational modes. Under such conditions, it is also possible for the distribution of molecules across the quantized vibrational energy states to not follow the equilibrium Boltzmann form. In addition, finite rate activation of the upper vibrational levels has an important coupling to the rate of molecular dissociation.

Computation of hypersonic flows in the continuum flow regime is performed using traditional Computational Fluid Dynamics (CFD). Under non-continuum conditions, the direct simulation Monte Carlo (DSMC) method is most commonly employed. Due to the importance of vibrational relaxation, a number of models have been developed for use in both CFD and DSMC flow simulations. A primary purpose of the present investigation is to assess the current status of the DSMC vibrational relaxation models.

This paper first describes low- and high-fidelity vibrational relaxation models used in CFD formulations. Next, the standard (low-fidelity) approach for DSMC is described. Subsequently, a high-fidelity model for DSMC, that is consistent with the high-fidelity CFD approach, is introduced. Equilibrium heat bath DSMC simulation results are presented to assess the consistency of the new model against the CFD version. Nonequilibrium heat bath studies are presented in heating and cooling environments to compare the new and standard DSMC vibrational relaxation models. Comparison of the models is further undertaken for one-dimensional, normal shock waves that include a case for which experimental data exist. The paper closes with a summary and final conclusions.

\footnotetext{
* Professor, Associate Fellow AIAA.

${ }^{\dagger}$ Senior Research Aerospace Engineer, Associate Fellow AIAA.
} 


\section{Vibrational Relaxation Models}

\section{A. Low-Fidelity CFD Approach}

For conditions close to equilibrium, a common description of the vibrational relaxation process is provided by the Landau-Teller equation [1]:

$$
\frac{d T_{v}}{d t}=\frac{T-T_{v}}{\tau_{v}}
$$

where $T_{v}$ is the vibrational temperature, $T$ is the translational temperature, and $\tau_{v}$ is the vibrational relaxation time. In state-of-the-art CFD codes, finite rate vibrational relaxation is almost always modeled in terms of vibrational energy:

$$
\frac{d \varepsilon_{v}}{d t}=\frac{\varepsilon_{v}^{*}-\varepsilon_{v}}{\tau_{v}}
$$

where $\varepsilon_{v}$ is the vibrational energy, $\varepsilon_{v}^{*}$ is the vibrational energy at the translational temperature, and $\tau_{v}$ is again the vibrational relaxation time that is usually evaluated as:

$$
\tau_{v}=\tau_{M W}+\tau_{P}
$$

with the first term due to Millikan and White [2] and the second term, a high temperature correction, due to Park [3], has the following form:

$$
\tau_{p}=\frac{1}{n \sigma_{v} C}
$$

where $n$ is the total number density, $C$ is the average thermal speed. Several forms for the vibrational cross section have been proposed from Park's original form

$$
\sigma_{v}=3 \times 10^{-21}\left(\frac{50,000}{T}\right)^{2}
$$

to a form derived from DSMC studies [4]:

$$
\sigma_{v}=5.81 \times 10^{-21}
$$

This low fidelity approach will be referred to as CFD-LT and it attempts to describe the detailed processes of actual vibrational relaxation that involves both vibration-translation (VT) and vibration-vibration (VV) energy transfer processes among quantized vibrational energy states using a single relaxation equation.

\section{B. High-Fidelity CFD Approach}

Adamovich et al. describe the development of vibrational transition rate coefficients based on the Forced Harmonic Oscillator (FHO) approach [5,6]. The rate coefficients are obtained through analytical, thermal averaging of collision-based transition probabilities. These probabilities are described in more detail in Section D and form the basis of a new, high fidelity DSMC model. Rate coefficient expressions are developed separately for vibrationtranslation (VT) and vibration-vibration (VV) transitions. The studies of Adamovich et al. [5,6] show that these models provide rate coefficients for nitrogen that are in good agreement with values obtained by Billing and Fisher using a quantum classical model [7]. The VT and VV rate coefficients may then be used in a Master Equation formulation to simulate the state-resolved vibrational relaxation process [8] that has been included in hypersonic flow field modeling [9].

\section{Low-Fidelity DSMC Approach}

Finite rate vibrational relaxation is usually modeled in the DSMC framework using a probability of vibrational energy exchange for each collision that is consistent at the macroscopic level with the CFD-LT approach [10]. For collisions that are selected for vibrational relaxation, a quantized form [11] of the Larsen-Borgnakke energy exchange model [12] is employed. Usually, the vibrational energy levels are described using a harmonic oscillator model. This low fidelity approach will be referred to as DSMC-LB and it acts to equilibrate the translational and vibrational energy modes of the molecules in the collisions selected for vibrational relaxation. 


\section{New, High-Fidelity DSMC Approach}

The basis for the new, high-fidelty DSMC model is the basic transition probability of the FHO model described by Adamovich et al. [6]. Specifically, in a molecule-molecule collision, the probability of transition from initial vibrational levels $\left(i_{1}, i_{2}\right)$ to final vibrational levels $\left(f_{1}, f_{2}\right)$ is given by:

$$
P_{V V T}\left(i_{1}, i_{2} \rightarrow f_{1}, f_{2}, \varepsilon, \rho\right)=\left|\sum_{r=0}^{n} C_{r+1, i_{2}+1}^{i_{1}+i_{2}} C_{r+1, f_{2}+1}^{f_{1}+f_{2}} \exp \left[-i\left(f_{1}+f_{2}-r\right) \rho\right] P_{V T}^{1 / 2}\left(i_{1}+i_{2}-r \rightarrow f_{1}+f_{2}-r, 2 \varepsilon\right)\right|^{2}(5 \mathrm{a})
$$

where

$$
P_{V T}(i \rightarrow f, \varepsilon)=i ! f ! \varepsilon^{i+f} \exp (-\varepsilon)\left|\frac{(-1)^{r}}{r !(i-r) !(f-r) !} \frac{1}{\varepsilon^{r}}\right|^{2}
$$

and

$$
\begin{gathered}
\varepsilon=S_{V T} \frac{4 \pi^{3} \omega\left(\tilde{m}^{2} / \mu\right) \gamma^{2}}{\alpha^{2} h} \sinh ^{-2}\left(\frac{\pi \omega}{\alpha \bar{v}}\right) \\
\rho=\left(S_{V V} \frac{\alpha^{2} \bar{v}^{2}}{\omega_{1} \omega_{2}}\right)^{1 / 2}
\end{gathered}
$$

In these equations: $C_{a, b}^{c}$ are transformation matrices defined in Ref. $6, S_{V T}$ and $S_{V V}$ are steric factors, $\omega$ is the oscillator frequency, $\tilde{m}$ is the collision reduced mass, $\mu$ is the oscillator reduced mass, $\gamma$ is oscillator mass ratio, $\alpha$ characterizes the intermolecular potential, $h$ is Planck's constant, and $\bar{v}$ is the symmetrized relative velocity. Further definitions and numerical values of the key parameters are provided in Ref. 6 . It should be noted that a number of prior DSMC studies have also considered state-resolved vibrational relaxation [13-16]. For example, in Ref. 16, the purely vibration-translation probability, Eq. (5b), was used to analyze vibrational relaxation processes. The present study represents a significant increase in fidelity by allowing full VVT transitions, and is referred to as the DSMC-FHO model. The vibrational levels are described by an anharmonic oscillator model in the DSMC-FHO model.

Due to the complicated mathematical form of Eqs. (5), the evaluation of transition probabilities for each collision in a DSMC computation is expensive numerically. Therefore, a table of probabilities is pre-computed for a fixed number of relative velocity bins. In the present work, the relative velocity bin size employed is $100 \mathrm{~m} / \mathrm{s}$, and transitions of up to \pm 5 quantum levels are considered. To further aid numerical performance, two different tables are generated. The first table provides the total probability of all transitions from the current vibrational levels of the two molecules in the collision. If it is determined that a transition does occur, a second table is then employed to decide, using an acceptance-rejection scheme, which of the transitions actually occurs. The tabulated approach is found to offer excellent agreement with more expensive DSMC computations in which the transitions are evaluated using the full formulae at a small fraction of the numerical cost.

\section{Results}

The primary purpose of this study is to develop and assess a high fidelity, state-resolved, vibrational relaxation model for the DSMC technique. This goal is achieved by performing a sequence of investigations that mainly consider molecular nitrogen including: an evaluation of the low-fidelity Larsen-Borgnakke DSMC model; FHO rate coefficient studies; nonequilibrium relaxation studies; shock wave studies, and studies involving carbon monoxide.

\section{A. Evaluation of the Low-Fidelity DSMC Model}

A heat bath is a zero dimensional configuration in which the energy modes of the gas are initialized to Boltzmann distributions specified by a mode temperature. For an equilibrium heat bath, used for rate coefficient evaluation, all mode temperatures are equal. For relaxation studies, the translational and rotational temperatures are equal, while the vibrational temperature is set either lower (vibrational heating) or higher (vibrational cooling). A vibrational heating relaxation study is first performed to compare the low-fidelity Larsen-Borgnakke model for DSMC (DSMC-LB) with the low-fidelity Landau-Teller (CFD-LT) and high-fidelity Forced Harmonic Oscillator (CFD-FHO) CFD models. Figures 1a shows the time evolution of the translational and vibrational temperatures obtained with the CFD-LT and DSMC-LB models for a nitrogen heat bath with initial conditions: $\mathrm{p}=101,325 \mathrm{~Pa}$, 
$\operatorname{Ttr}=10,000 \mathrm{~K}, \mathrm{Tv}=1,000 \mathrm{~K}$. Clearly, the two approaches offer almost identical relaxation profiles. However, when the vibrational energy distribution functions (VEDFs) from these simulations are compared, see Fig. 1b, it is clear that while the CFD-LT approach assumes Boltzmann distributions at the vibrational temperature, the DSMC-LB model predicts strongly nonequilibrium distributions. The DSMC-LB model essentially predicts that the molecules consist of two distinct populations given by the initial cold temperature and the final equilibrium temperature. This is not surprising as the basic concept of the DSMC-LB model is to instantly equilibrate the internal modes of a very small fraction of all collisions. Similar bi-modal vibrational energy distributions have been seen in previous DSMC studies $[14,16,17]$, and one of the primary goals of the present study is to try and determine whether this behavior is physically accurate. Figure 1c re-plots the DSMC-LB data using vibrational energy as the variable and the straight lines are Boltzmann distributions based on the vibrational temperatures computed in the simulation. The nonequilibrium behavior of the DSMC-LB model in Fig. 1c can be compared with the profiles shown in Fig. 1d that are obtained using the CFD-FHO model including both VT and VV transitions up to five quantum steps. Clearly, the CFD-FHO model predicts a significantly smaller degree of nonequilibrium for the vibrational relaxation process.

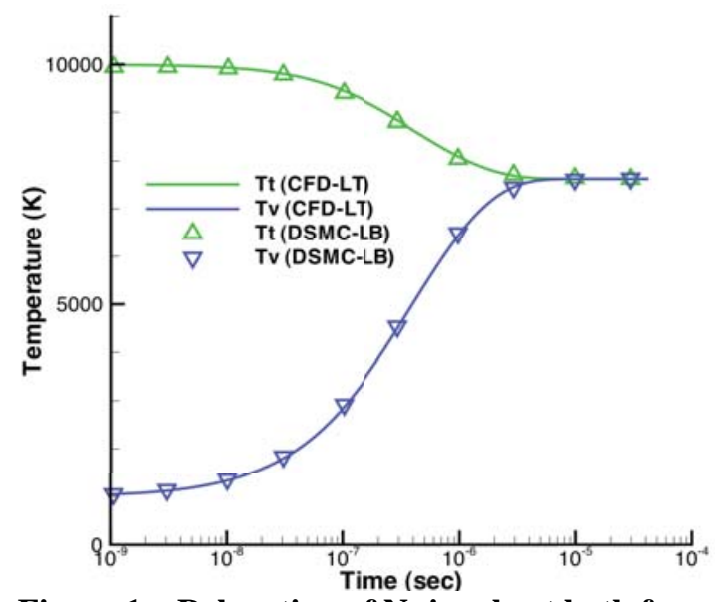

Figure 1a. Relaxation of $\mathrm{N}_{2}$ in a heat bath for a vibrational heating environment.

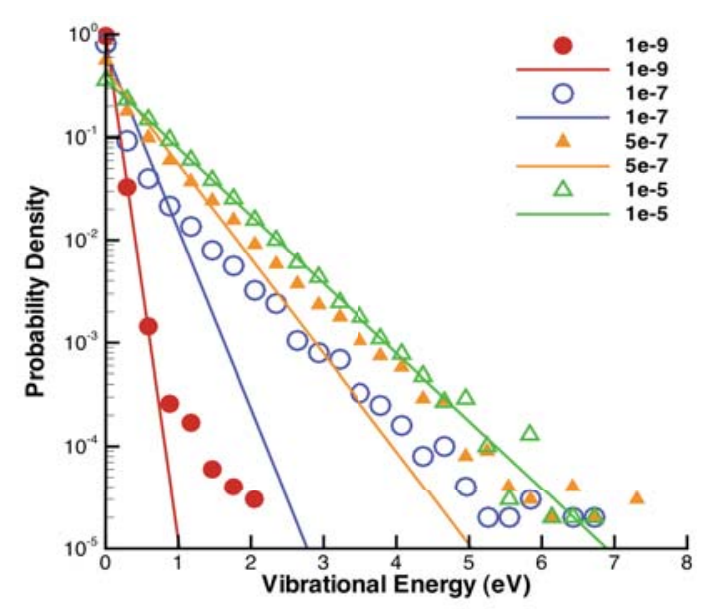

Figure 1c. Time evolution of the VEDF: lines=Boltzmann; symbols=DSMC - LB.

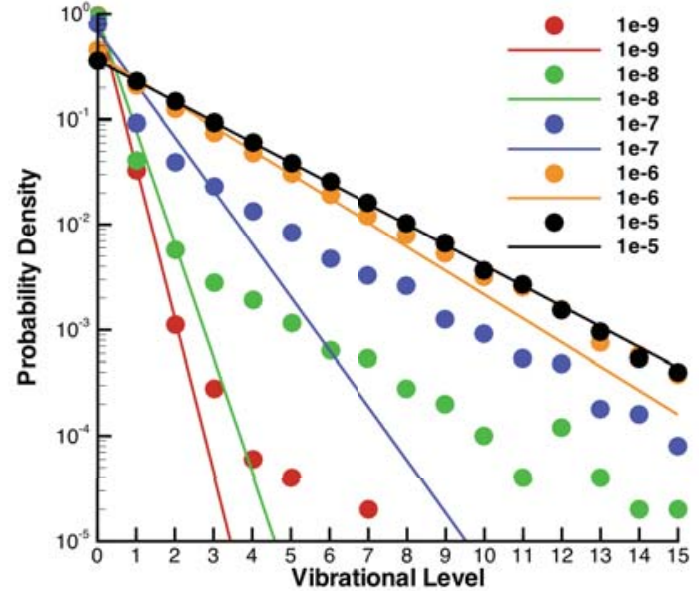

Figure 1b. Time evolution of the VEDF: lines=CFDLT; symbols=DSMC-LB.

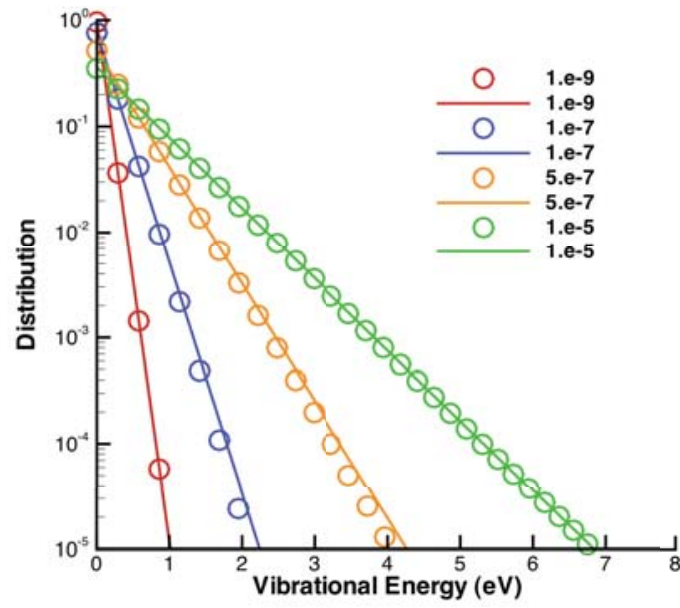

Figure 1d. Time evolution of the VEDF: lines=Boltzmann; symbols=CFD-FHO.

Since a heath bath is a zero dimensional case without flow, it is useful to consider the performance of the DSMC-LB model in a one-dimensional shock wave to determine whether the highly nonequilibrium vibrational energy distribution is again predicted. Using nitrogen as the test gas again, a computation is performed at Mach 10 matching the highest Mach number flow condition of the experiments performed by Alsmeyer [18]. Alsmeyer used an electron beam fluorescence technique to measure the density profiles through a series of normal shock waves. It 
is stated in his study that vibrational relaxation effects are not important even at Mach 10, and we use this opportunity to assess that statement. Figure 2a shows profiles of density $(\rho)$, and the temperatures associated with the translational $(\mathrm{Tt})$, rotational $(\mathrm{Tr})$, and vibrational $(\mathrm{Tv})$ modes. The profiles are normalized using the same approach as Alsmeyer. The solid line profiles represent results in which vibrational relaxation is included in the analysis whereas the dotted lines with circles are obtained from a simulation that omits vibrational relaxation. The excellent agreement between the two sets of profiles confirms Alsmeyer's statement that his experimental measurements of the shock structure are not greatly affected by the vibrational relaxation process, even at Mach 10. Of course, the profiles far downstream of the shock front are significantly influenced by the vibrational activation process. Figure $2 \mathrm{~b}$ shows the evolution of the vibrational energy distribution function through the shock wave. These distributions are very similar to those predicted by the DSMC-LB approach shown in Fig. $1 \mathrm{~b}$ for the heat bath environment. It is therefore concluded that the prediction by the DSMC-LB model of strongly nonequilibrium vibrational energy distributions is a general result that needs to be checked against a high fidelity DSMC model.

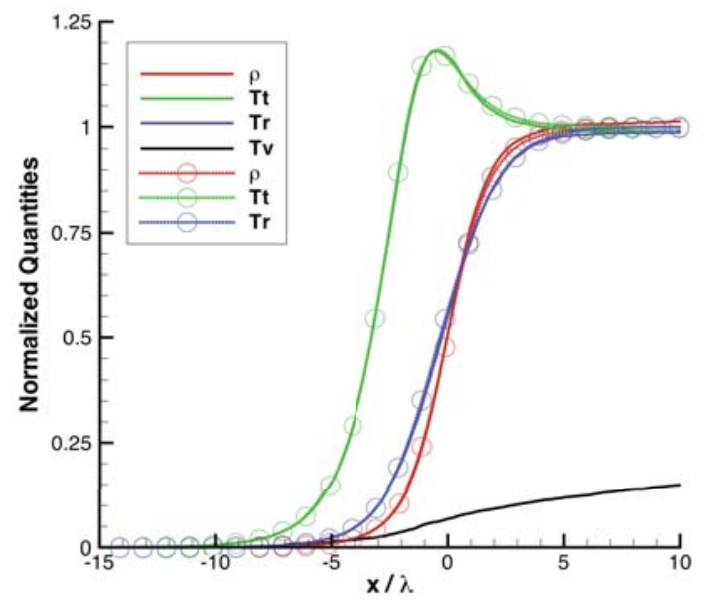

Figure 2a. Profiles at the front of a Mach 10 normal shock wave in nitrogen: symbols=vibration omitted

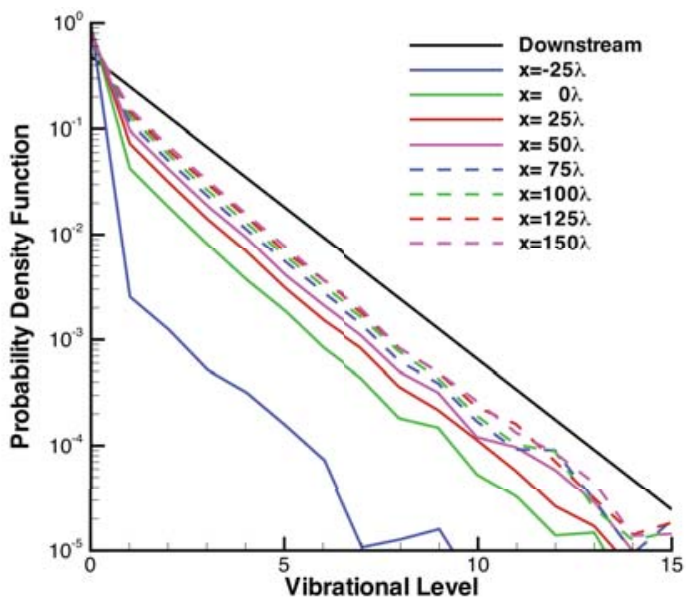

Figure 2b. Spatial evolution of the vibrational energy distribution function in the Mach 10 shock.

\section{B. Assessment of the High-Fidelity DSMC Model}

The prior results indicate that the low-fidelity DSMC-LB model predicts a degree of thermal nonequilibrium in both heat bath and shock wave studies that is much stronger than that predicted by the high-fidelity CFD-based FHO model. This finding motivates the development of the high-fidelity DSMC-FHO model described earlier. In the present section, equilibrium heat bath studies are first performed using DSMC-FHO in which the transition probabilities are integrated over all collisions to determine the transition rates. All values of the FHO parameters employed in Ref. 6 for $\mathrm{N}_{2}-\mathrm{N}_{2}$ transitions are employed in the DSMC-FHO model except for the steric factors that are set to: $S_{V T}=1 / 9$ and $S_{V V}=1 / 40$ (compared to $S_{V T}=1 / 2$ and $S_{V V}=1 / 25$ used in Ref. 6). The rates obtained from DSMC-FHO are compared in Figs. 3 with published data for the CFD-FHO model [6] as well as to results obtained using a quantum classical method by Billing and Fisher [7]. In general, the rates evaluated using the DSMC model are in good agreement with the CFD-FHO model of Adamovich et al. [6] which provides some validation of the approximations employed in the development of the CFD-FHO approach. The fact that different steric factors are required to achieve good agreement between these two FHO models suggests that the CFD-FHO approximations mainly result in an inaccuracy that can be accounted for through a multiplicative constant. The comparisons of the DSMC-FHO model with the detailed computations of Billing and Fisher [7] are also satisfactory including the VV transition rates (Fig. 3d). 


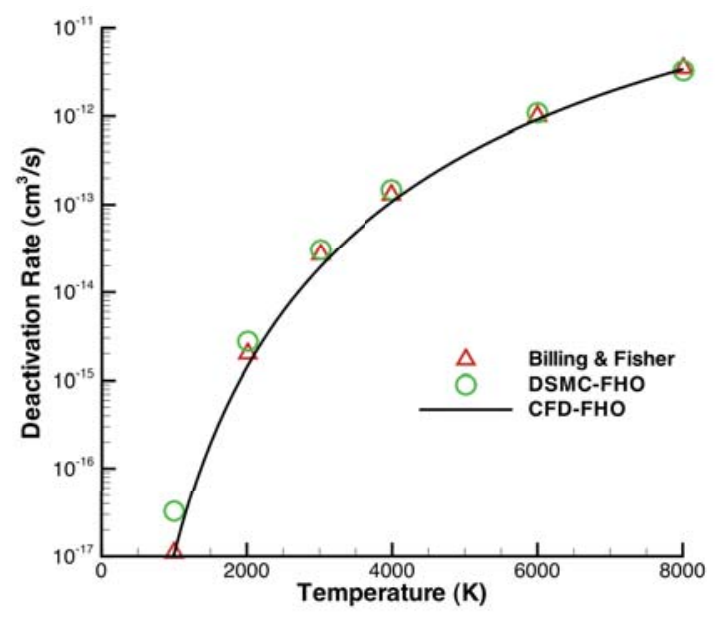

Figure 3a. VT de-excitation rate $(1,0)$ to $(0,0)$ as a function of temperature.

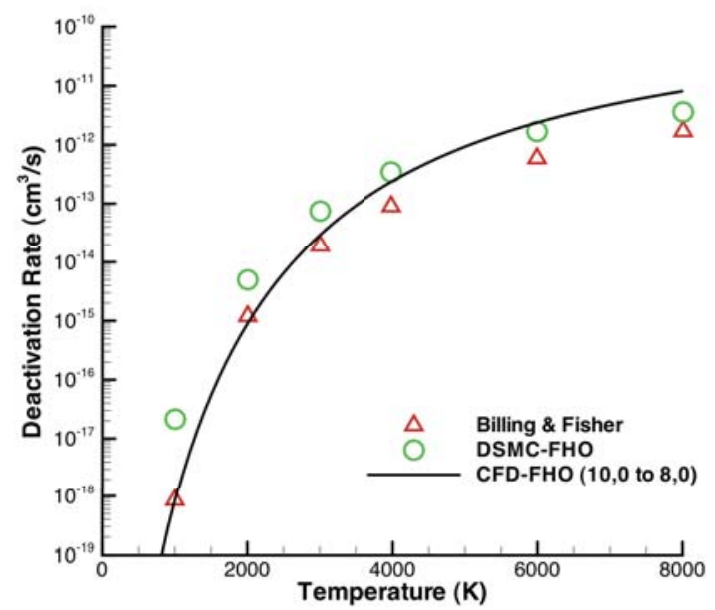

Figure 3c. VT de-excitation rate $(10,5)$ to $(8,5)$ as a function of temperature.

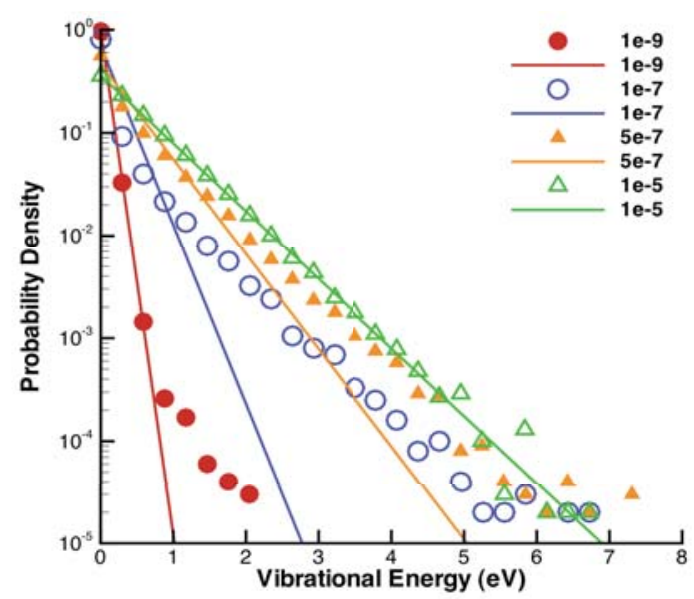

Figure 4a. Time evolution of the VEDF: lines=Boltzmann; symbols=DSMC-LB.

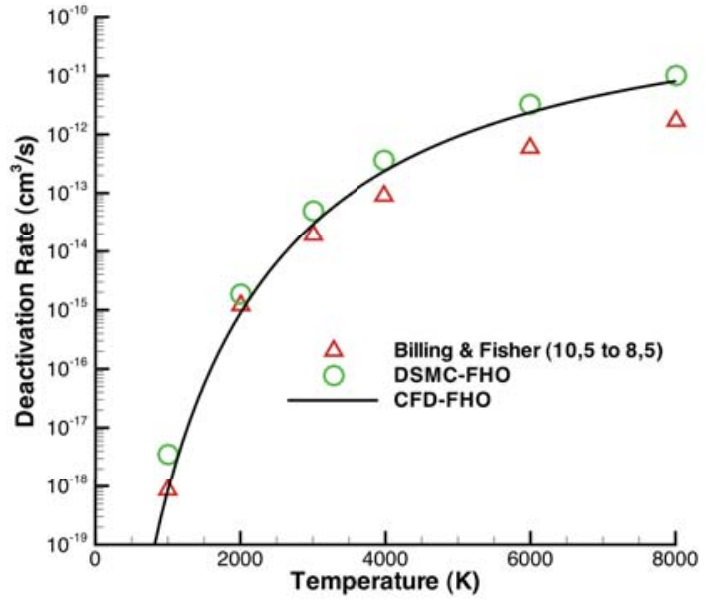

Figure 3b. VT de-excitation rate $(10,0)$ to $(8,0)$ as a function of temperature.

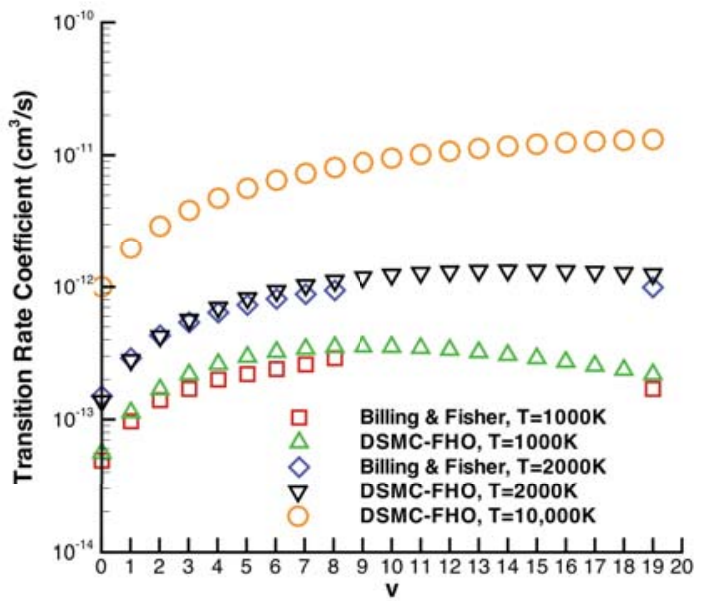

Figure 3d. VV transition rate $(v, 1)$ to $(v+1,0)$ as a function of vibrational level and temperature.

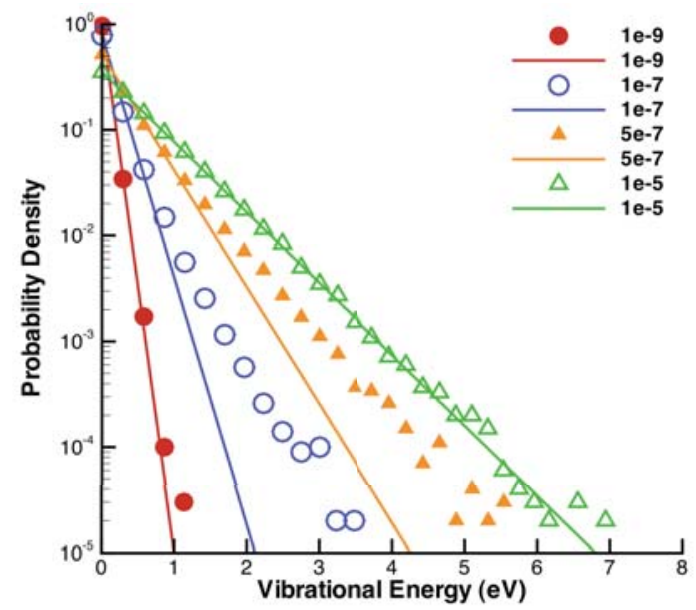

Figure 4b. Time evolution of the VEDF: lines=Boltzmann; symbols=DSMC-FHO. 
Having established that the DSMC-FHO model produces transition rates consistent with the quantum classical model, the DSMC model is now applied to the same kind of configurations studied earlier. Figure 4a is a repeat of Fig. 1c included here to facilitate comparison to Fig. 4b that shows the time evolutions obtained with the DSMC-FHO model of the vibrational energy distributions for the same vibrational heating conditions studied in Figs. 1. While the DSMC-FHO model still displays some nonequilibrium, it is significantly less bi-modal in nature than the distributions predicted by the DSMC-LB model.

Figures $5 \mathrm{a}$ and $5 \mathrm{~b}$ show the temperature profiles and vibrational energy distribution evolution for a vibrational cooling case for initial conditions: $\mathrm{p}=101,325 \mathrm{~Pa}, \mathrm{Ttr}=3,000 \mathrm{~K}, \mathrm{Tv}=10,000 \mathrm{~K}$. In Fig. 5a, results are provided from all four models, both low- and high-fidelity approaches using CFD and DSMC. The results show that the two lowfidelity models (CFD-LT and DSMC-LB) agree with each other and they predict a significantly slower relaxation rate than the two high-fidelity models (CFD-FHO and DSMC-FHO) that again agree with each other. Figure 5b shows the evolution of the vibrational energy distribution obtained with the DSMC-LB model. Careful inspection of the distribution shows that once again it is composed of two populations of molecules: one defined by the initial temperature and the second defined by the final equilibrium temperature. Figure $5 \mathrm{c}$ shows the equivalent profiles obtained with the DSMC-FHO model. To a very good approximation, the FHO model predicts that the relaxation process occurs through a sequence of near-Boltzmann distributions, and so once again this model produces a smaller degree of nonequilibrium in comparison to the low-fidelity DSMC-LB approach.

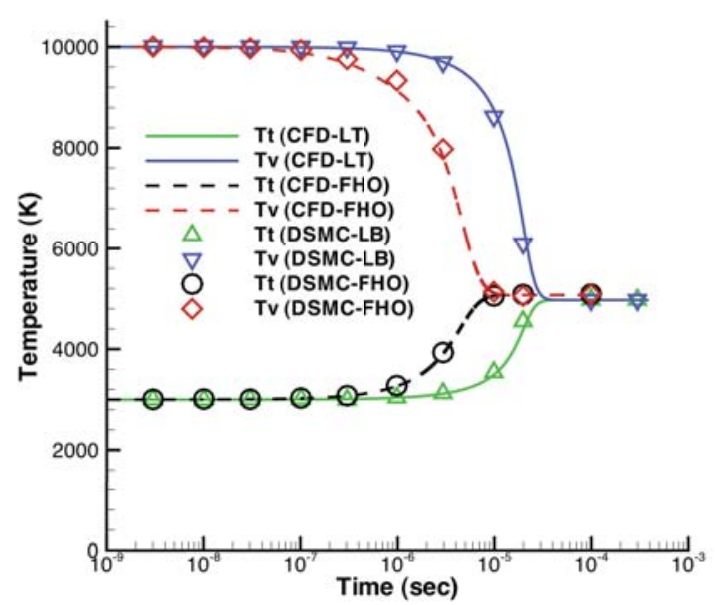

Figure 5a. Relaxation of $\mathbf{N}_{2}$ in a heat bath for a vibrational cooling environment.

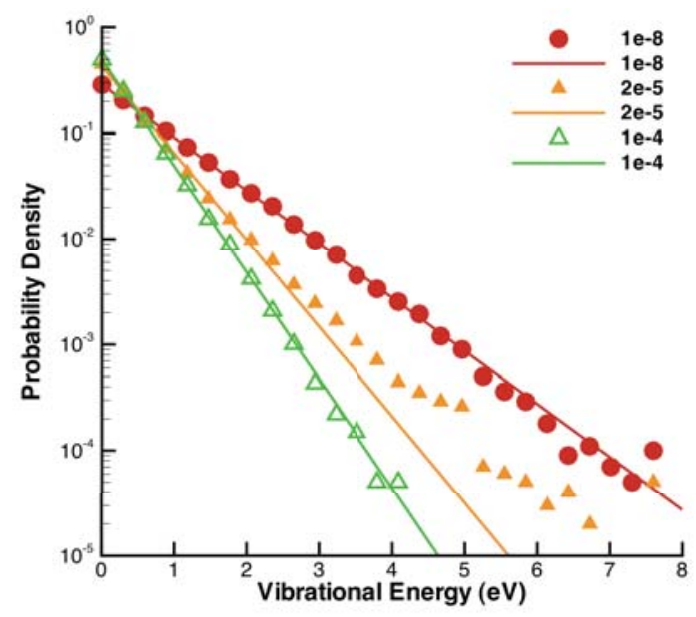

Figure 5b. Time evolution of the VEDF: lines=Boltzmann; symbols=DSMC-LB.

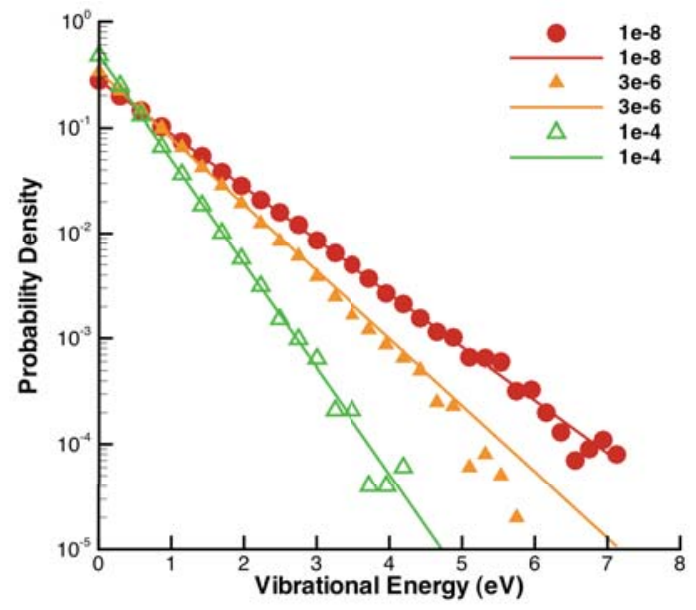

Figure 5c. Time evolution of the VEDF: lines=Boltzmann; symbols=DSMC-FHO.

The DSMC-FHO model is next applied to Alsmeyer's Mach 10 nitrogen shock wave discussed earlier. While the temperature and density profiles are very similar to those obtained with the DSMC-LB model, there are 
significant differences in the vibrational energy distribution functions, shown in Figs. $6 \mathrm{a}$ and $6 \mathrm{~b}$. In particular, the distributions obtained with the DSMC-FHO model, in Fig. 6b, again show a significantly reduced level of vibrational nonequilibrium in comparison to the low-fidelity LB approach.

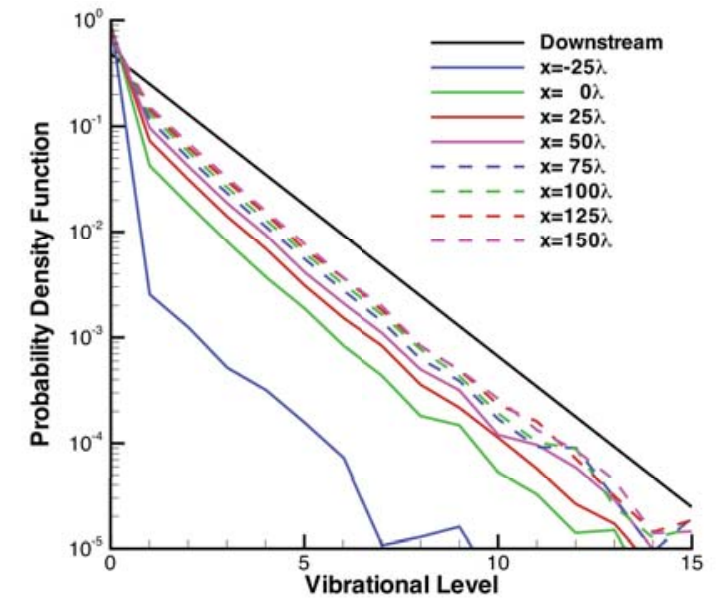

Figure 6a. Spatial evolution of the VEDF in the Mach 10 shock: DSMC-LB.

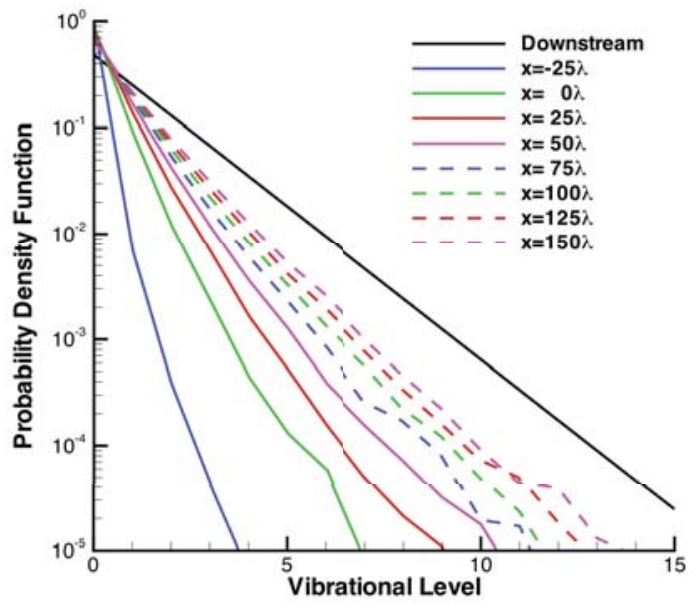

Figure 6b. Spatial evolution of the VEDF in the Mach 10 shock: DSMC-FHO.

\section{Carbon Monoxide Studies}

Unfortunately, no experimental measurements have been performed in molecular nitrogen to evaluate the differences predicted in vibrational energy distributions by the DSMC-LB and DSMC-FHO models that are shown in Figs. 6. One set of experimental data that does appear to offer the potential to assess the DSMC vibrational relaxation models is the study performed by Meolans and Brun [19] who measured relative vibrational populations in strong shock waves of carbon monoxide. Parameters employed in the DSMC-FHO model for CO are: $\alpha=3.6 \times 10^{10} \mathrm{~m}^{-1}, S_{V T}=1 / 9$ and $S_{V V}=3 / 40$. In order to apply the DSMC-FHO model to CO, it is of course necessary to repeat the equilibrium transition rate coefficient studies. Examples of comparisons between the DSMC integrated FHO rates, rates evaluated using the CFD-FHO formulae, and semi-classical calculations of Cacciatore and Billing [20] are shown in Figs. 7a and 7b. Once again, the agreement between the DSMC-FHO and CFD-FHO results is good. Agreement with the semi-classical calculations is less satisfactory.

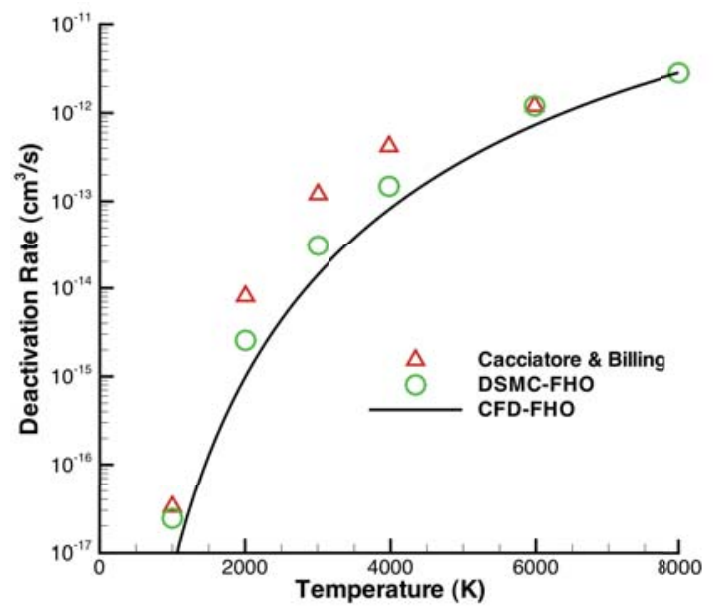

Figure 7a. VT de-excitation rate $(1,0)$ to $(0,0)$ as a function of temperature for $\mathrm{CO}$.

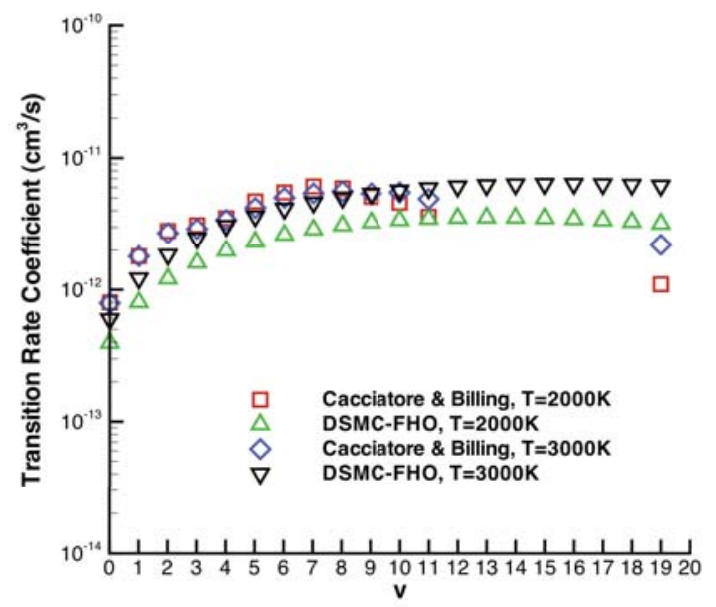

Figure $7 \mathrm{~b}$. VV transition rate $(\mathrm{v}, 1)$ to $(\mathrm{v}+1,0)$ as a function of temperature for $\mathrm{CO}$.

Finally, the DSMC-LB and the DSMC-FHO models are applied to the conditions of the Mach 7 carbon monoxide shock wave studied by Meolans and Brun [19]. The transient profiles of the relative population of the $\mathrm{v}=4$ vibrational state obtained with the two DSMC models are compared with the experimental measurements in 
Figs. 8a and 8b. In Figs. 8, two results from each DSMC simulation are provided. The profile labeled "DSMC" is the actual result from the simulation for the relative population in the $v=4$ vibrational state. The profile labeled "DSMC-Boltzmann" is the relative population in $\mathrm{v}=4$ determined using the computed vibrational temperature along with an assumption of a Boltzmann energy distribution. Comparison of these two profiles provides an indication of the degree of vibrational nonequilibrium predicted by the two models. In Fig. 8a, the DSMC-LB model predicts a strong degree of vibrational nonequilibrium in the early stages of the vibrational activation process. The actual population predicted by the model is significantly higher than the Boltzmann assumption that is in good agreement with the experimental data in this region of the shock. This behavior is consistent with the nitrogen results reported earlier in this study and is perhaps the most significant finding of the current investigation. Namely, that the DSMCLB model appears to over-predict the populations of all but the lowest vibrational levels. By comparison, as shown in Fig. 8b, the two DSMC profiles obtained with the DSMC-FHO model are in good agreement with each other and with the experimental measurements. This result indicates, at least for carbon monoxide, that the high-fidelity DSMC-FHO model is able to simulate the state-resolved vibrational relaxation process more accurately than the low-fidelity DSMC-LB approach.
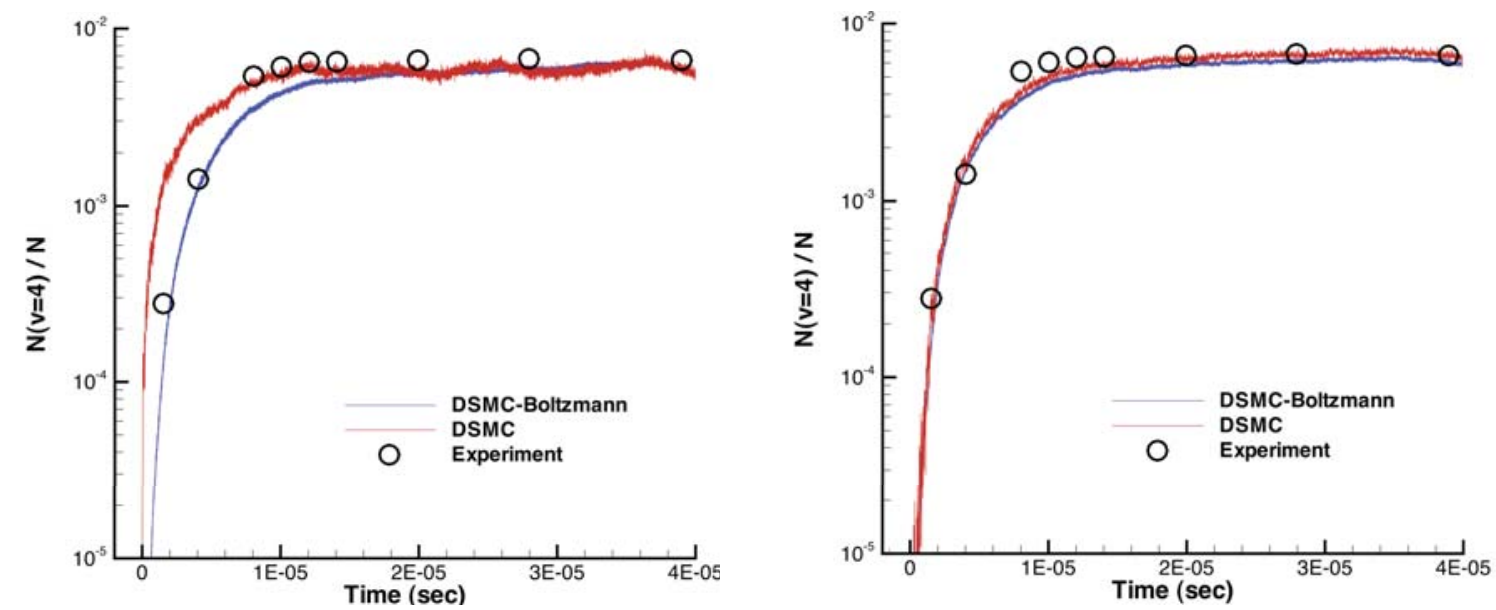

Figure 8a. Profiles of the relative population of $v=4$ Figure $8 \mathrm{~b}$. Profiles of the relative population of $v=4$ for a Mach 7 shock wave of CO: DSMC-LB.

\section{Summary and Conclusion}

High fidelity, state-resolved, vibrational relaxation models have been developed in the past for use in CFD computations of hypersonic flows. By comparison, the level of vibrational relaxation modeling using the DSMC technique has not been as sophisticated. In the present study, a high fidelity, state-resolved, vibrational relaxation model for the DSMC technique has been developed, implemented with numerical efficiency, verified to produce expected rate coefficients, and partly validated through its application to a Mach seven shock wave for which experimental measurements of the vibrational levels exist in the literature.

Future work will aim to extend the DSMC-FHO model to include the effects of molecular dissociation. In addition, in the present work, it was not possible to analyze the behavior of the highest vibrational levels due to statistical fluctuations. It is required to employ a numerical weighting scheme in order to track molecules in populations that occur with very small probability. Finally, it is extremely important for the further development of high fidelity physical models for both CFD and DSMC that experiments be performed in air species using advanced, optical diagnostics, under nonequilibrium flow conditions, similar to the shock tube experiment in carbon monoxide that was employed in the current study.

\section{Acknowledgments}

The authors gratefully acknowledge the contributions made to this study by Igor Adamovich. Funding for IB was provided by the AFRL summer faculty research program. EJ acknowledges support by a contract from the U.S. Air Force Office of Scientific Research monitored by Fariba Fahroo. 


\section{References}

[1] Landau, L. and Teller, E., "Theory of Sound Dispersion," Physics Sowjetunion, Vol.10, 1936, pp. 34-43.

[2] Milliken, R.C. and White, D.R., "Systematics of Vibrational Relaxation," Journal of Chemical Physics, Vol. 39, 1963, pp. 3209-3213.

[3] Park, C., "Review of Chemical Kinetic Problems of Future NASA Missions, I: Earth Entries," Journal of Thermophysics and Heat Transfer, Vol. 7, 1993, pp. 385-398.

[4] Haas, B.L. and Boyd, I.D., "Models for Direct Monte Carlo Simulation of Coupled Vibration-Dissociation," Physics of Fluids A, Vol. 5, 1993, pp. 478-489.

[5] Adamovich, I.V., Macheret, S.O., Rich, J.W., and Treanor, C.E., "Vibrational Relaxation and Dissociation Behind Shock Waves Part 1: Kinetic Rate Models," AIAA Journal, Vol. 33, 1995, pp. 1064-1069.

[6] Adamovich, I.V., Macheret, S.O., Rich, J.W., and Treanor, C.E., "Vibrational Energy Transfer Rates Using a Forced Harmonic Oscillator Model," Journal of Thermophysics and Heat Transfer, Vol. 12, 1998, pp. 57-65.

[7] Billing, G.D. and Fisher, E.R., "VV and VT Rate Coefficients in N2 by a Quantum-Classical Model," Chemical Physics, Vol. 43, 1979, pp. 395-401.

[8] Adamovich, I.V., Macheret, S.O., Rich, J.W., and Treanor, C.E., "Vibrational Relaxation and Dissociation Behind Shock Waves Part 2: Master Equation Modeling," AIAA Journal, Vol. 33, 1995, pp. 1070-1075.

[9] Josyula, E., "Computational Study of Vibrationally Relaxing Gas Past Blunt Body in Hypersonic Flows," Journal of Thermophysics and Heat Transfer, Vol. 14, 2000, pp. 18-26.

[10] Boyd, I.D., "Analysis of Vibration-Dissociation-Recombination Processes Behind Strong Shock Waves of Nitrogen," Physics of Fluids A, Vol. 4 (1), 1992, pp. 178-185.

[11] Bergemann, F. and Boyd, I.D., "New Discrete Vibrational Energy Method for the Direct Simulation Monte Carlo Method," Rarefied Gas Dynamics, Progress in Astronautics and Aeronautics, AIAA, Washington, Vol. 158, 1994, p. 174.

[12] Borgnakke, C. and Larsen, P.S., "Statistical Collision Model for Monte Carlo Simulation of Polyatomic Gas Mixture," Journal of Computational Physics, Vol. 18, 1975, pp. 405-420.

[13] Abe, T., "Inelastic Collision Model for Vibrational-Translational and Vibrational-Vibrational Energy Transfer in the Direct Simulation Monte Carlo Method," Physics of Fluids, Vol. 6, 1994, pp. 3175-3179.

[14] Wysong, I.J., Wadsworth, D.C., Weaver, D.P., and Campbell, D.H., "Influence of Vibrational Nonequilibrium on Chemically Reacting Rarefied Flows - Toward Experimental Verification of DSMC Models," AIAA Paper 19962023, June 1996.

[15] Wadsworth, D.C. and Wysong, I.J., "Vibrational Favoring Effect in DSMC Dissociation Models," Physics of Fluids, Vol. 9, 1997, pp. 3873-3884.

[16] Vijayakumar, P., Sun, Q. and Boyd, I.D., "Detailed Models of Vibrational-Translational Energy Exchange for the Direct Simulation Monte Carlo Method," Physics of Fluids, Vol. 11, 1999, pp. 2117-2126.

[17] Gimelshein, S.F., Boyd, I.D., Sun, Q., and Ivanov, M., "DSMC Modeling of Vibration-Translation Energy Transfer in Hypersonic Rarefied Flows," AIAA Paper 99-3451, June 1999.

[18] Alsmeyer, H., "Density Profiles in Argon and Nitrogen Shock Waves Measured By Absorption of an Electron Beam," Journal of Fluid Mechanics, Vol. 74, 1976, pp. 497-513.

[19] Meolans, J.G. and Brun, R., "Nonequilibrium Vibrational Population Behind Shock Waves: Comparison Theory - Experiments," Proceedings of the 15th International Symposium on Rarefied Gas Dynamics, Teubner, Stuttgart, 1986, p. 345.

[20] Cacciatore, M. and Billing, G.D., "Semiclassical Calculation of VV and VT Rate Coefficients in CO," Chemical Physics, Vol. 58, 1981, pp. 395-407. 